$1 \pi$

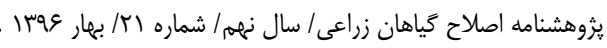

اثر يرتو تاما بر ميزان رشد و تعيين دز مناسب به منظور افزايش تنوع زنتيكى در

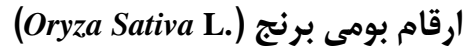

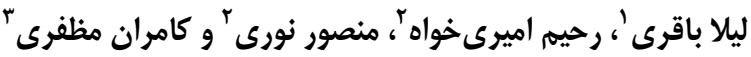

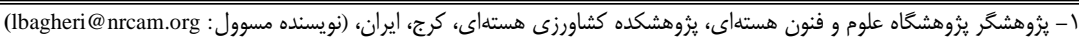

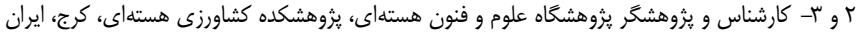

تاريخ دريافت:

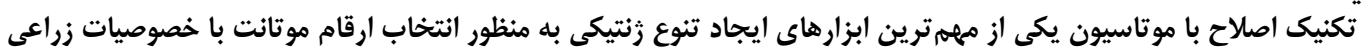

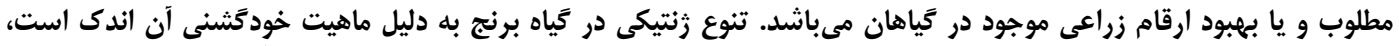

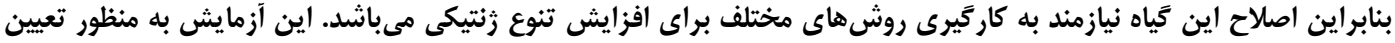

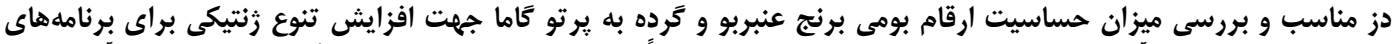

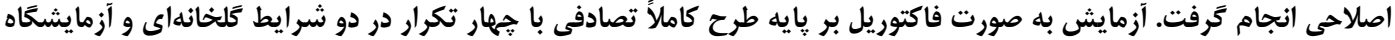

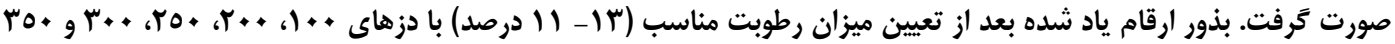

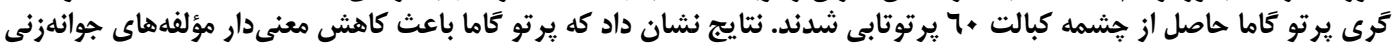

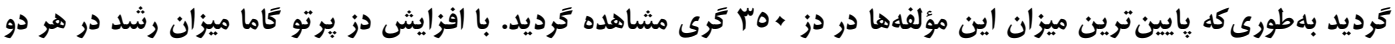

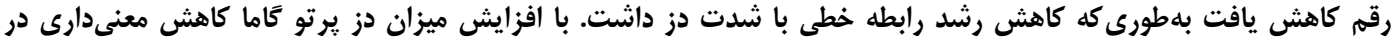

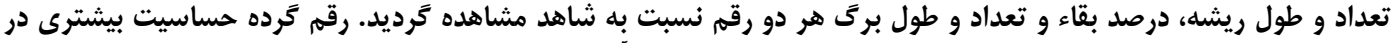

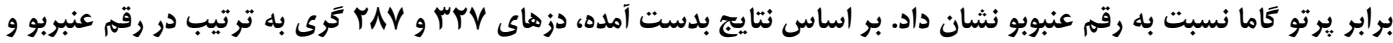

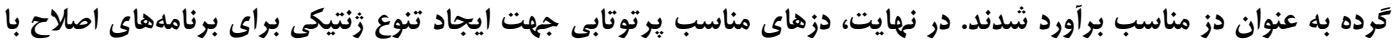

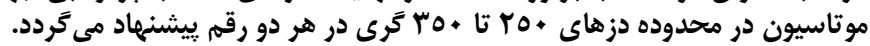

وازههاى كليدى: يرتو تاما، برنج، تنوع زنتيكى، موتاسيون

مشخص شود. براى تعبين بهترين دز جهت يرتو تور تابى بايد به

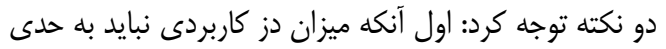

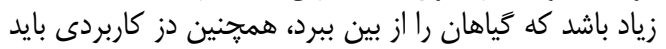

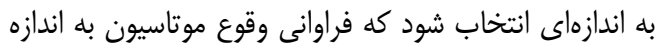

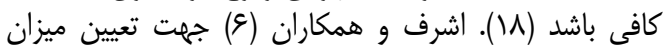

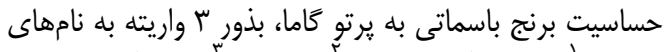

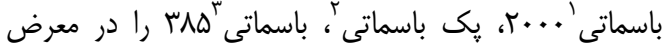

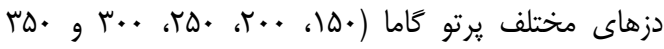

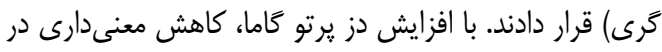

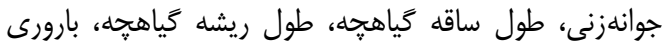

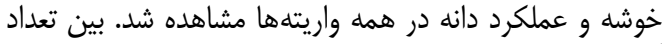

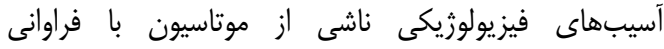

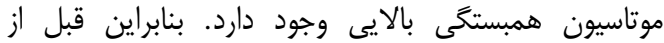

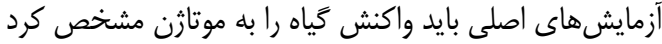

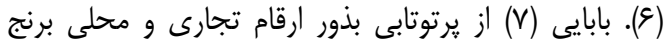

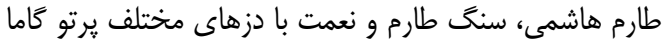

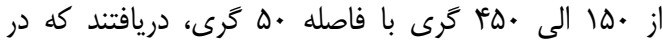

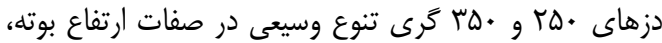

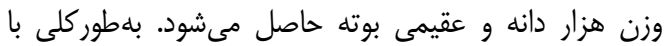

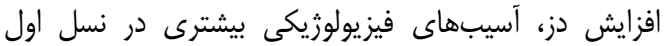

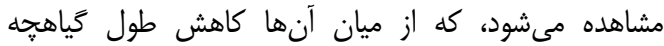

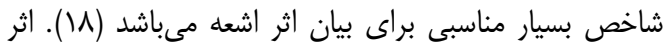

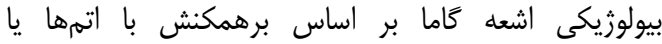

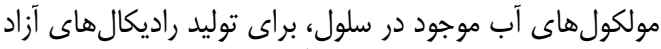

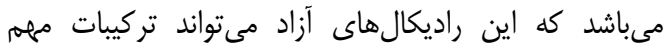

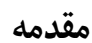

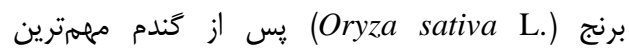

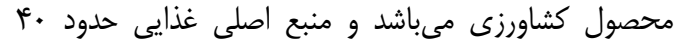

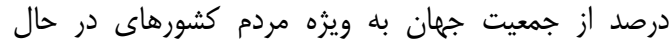

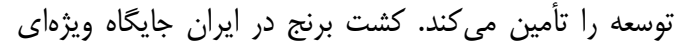

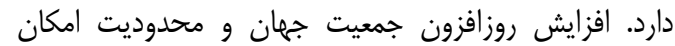

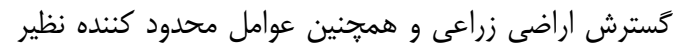

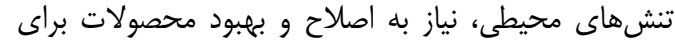

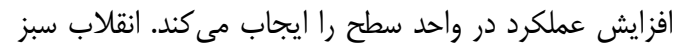

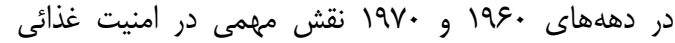

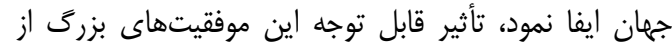

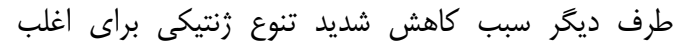

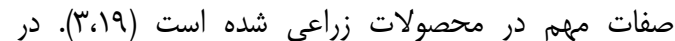

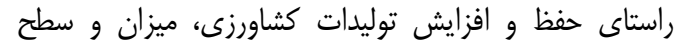

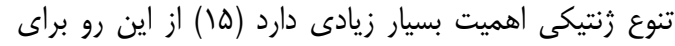

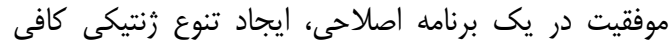

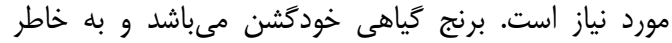

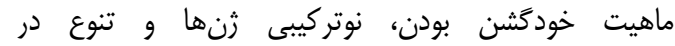

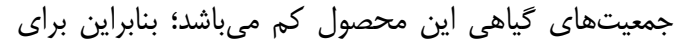

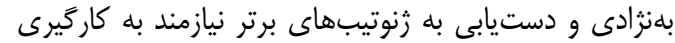

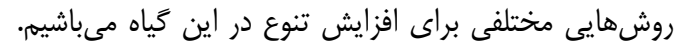

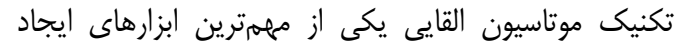

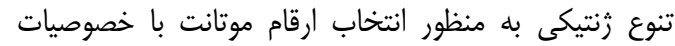

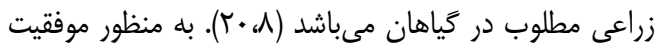
در يك برنامه اصلاحى به كمك موتاسيون بايد ابتدا دز مناسب 


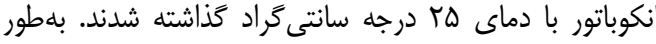

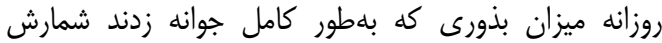
كرديد. سرعت جوانهزنى بذور از طرئ بريق رابط جرانه ذيل محاسبه

كرديد (1): (1): (1)

رابطه (1)

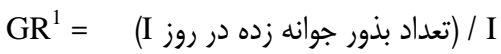

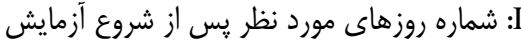

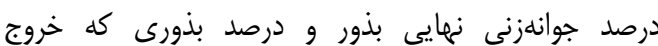

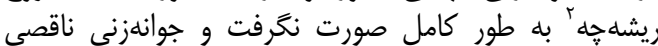

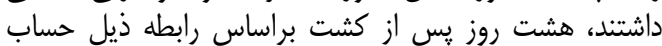

كر كريد (1) (1):

رابطه (T) (I) (1)

FGP= بذور جوانه زده تا بايان آزمايش)

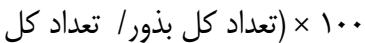

FGP

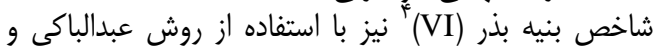
همكاران (T)، طبق فرمول زير محاسبه كَرديد

رابطه (广)

VI = ميانخين طول كياهجه (ميلى متر))

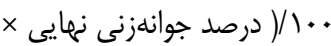

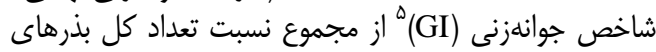

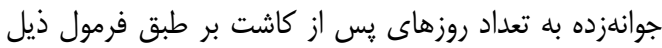

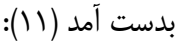

رابطه (i)

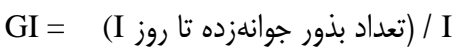

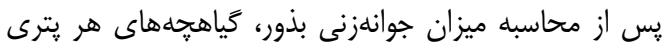

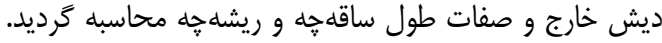

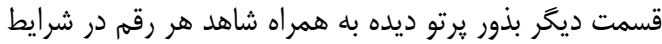

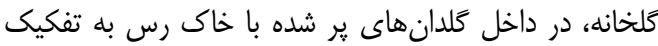

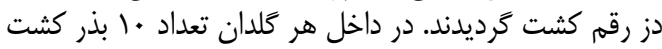

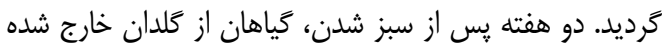

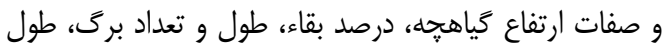

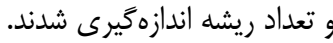

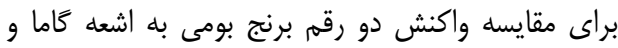
تعيين ميزان حساسيت آنها از شاخص حساسيت به به تنش

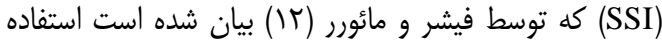

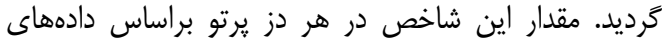

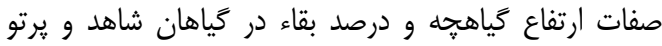
ديده براى هر رقم از رابطه ذيل محاسبه كرديد.

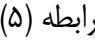

شاخص حساسيت به تنش (Stress Susceptibility Index)

$$
\mathrm{SI}=1 \frac{\overline{Y_{S}}}{\overline{Y_{p}}} \quad \mathrm{SSI}=\frac{1-\frac{Y_{S}}{Y_{p}}}{S I}
$$

شدت تنش، YP= SI = واكنش رقم مورد نظر در محيط بدون

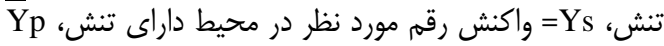

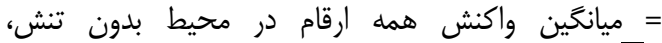
ميانگين واكنش همه ارقام در محيط داراى تنش است.

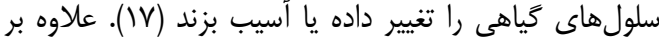

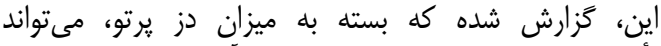

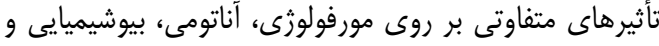

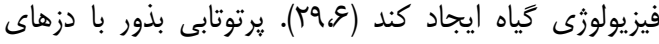

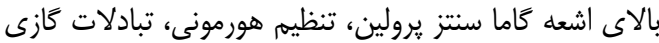

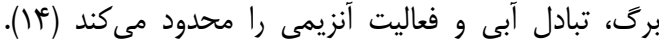

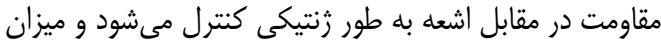

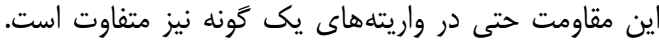

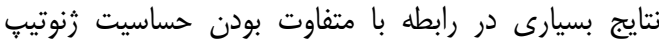

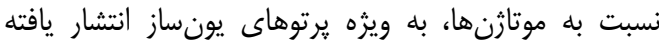

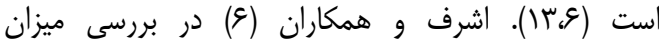

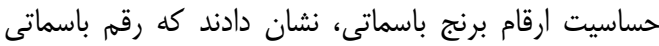

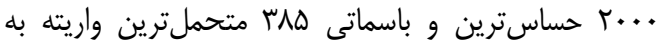

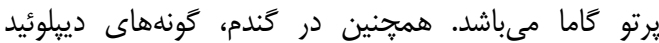

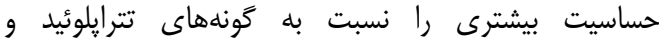

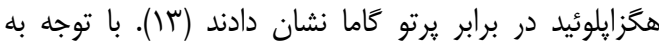

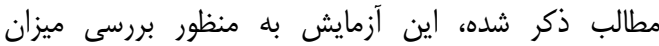

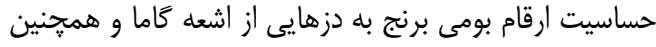
تعيين دز مناسب جهت ايجاد تنوع زنتيكى در اين ارقام إقام صورت

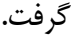

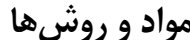

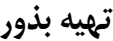

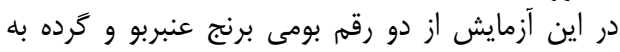

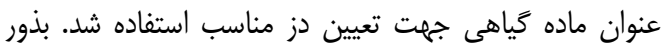

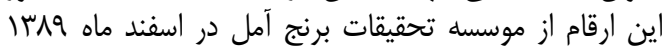

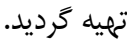
تنظيم رطوبت بذور

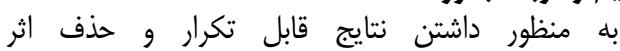

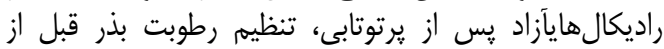

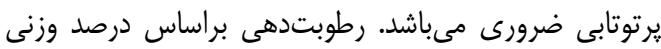

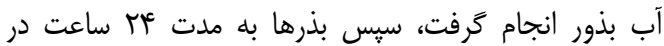
دماى

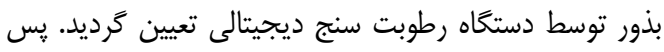

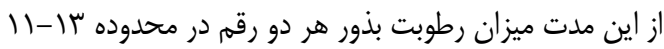

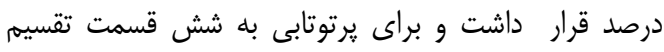

شدند.

يرتوتابى، كاشت و اندازهيرى صفات ضرورى

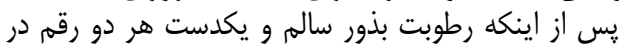

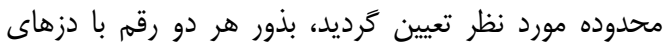

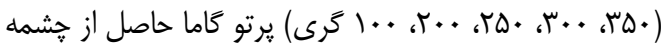

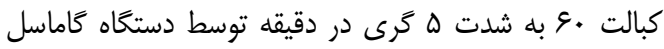

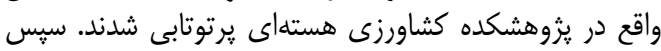

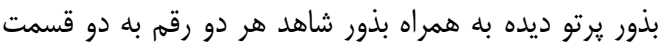

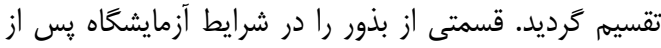

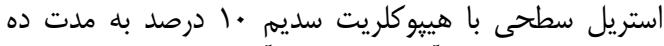

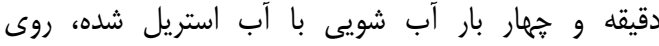

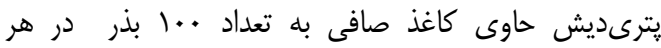

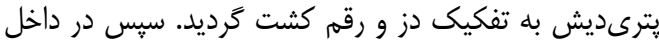

3- Final germination percentage

4- Vigor Index 
احتمال يك درصد اختلاف معنىدارى نشان داد (جدول ()). در

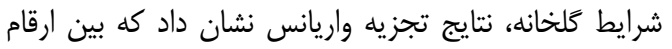

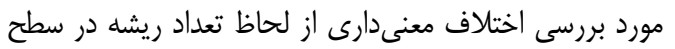

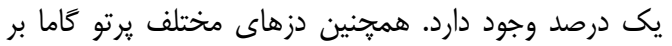

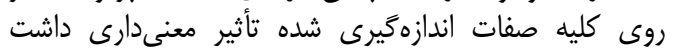

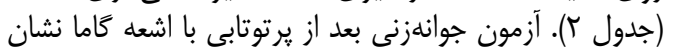

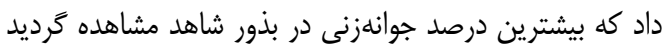

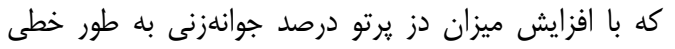

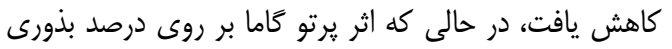

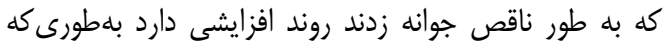

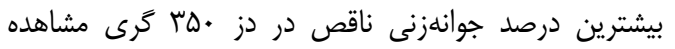

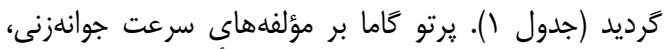

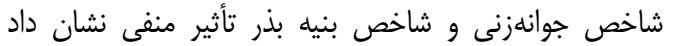

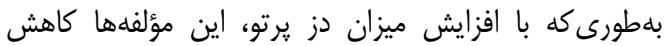

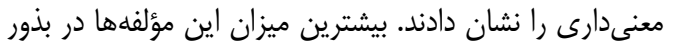

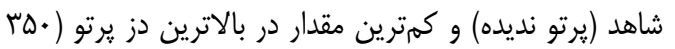

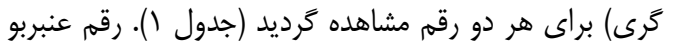

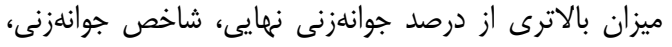

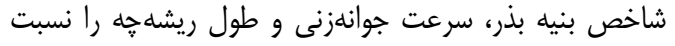

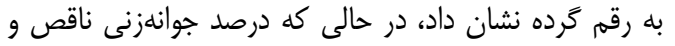

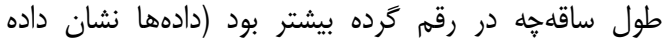

نشدند).
مقدار كمتر شاخص حساسيت به تنش نشاندهنده تغييرات

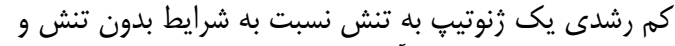

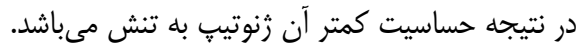
تجزيل أمارى

آزمايش در قالب طاري طرح فاكتوريل با دو سطح زنوتيت

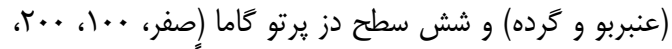

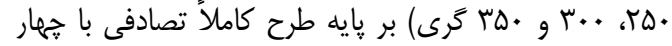

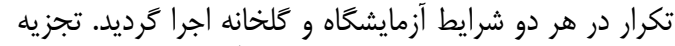

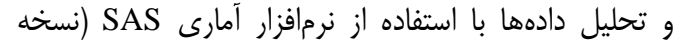

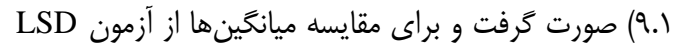

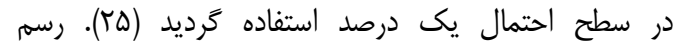

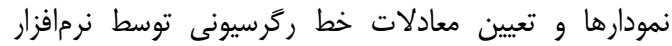
Excel

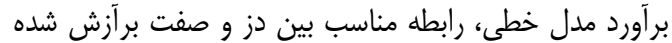
و دز مناسب تعيين گرديد.

\section{نتايج و بحث}

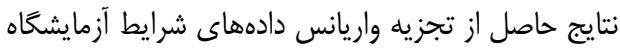

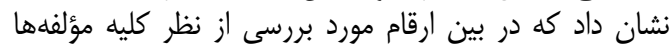

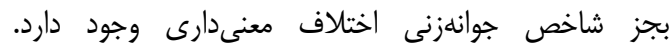

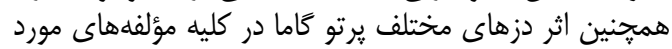

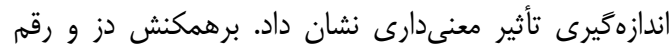

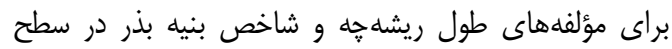

جدول ا- تأثير دزهاى مختلف يرتو كاما بر خصوصيات جوانهزنى ارقام برنج بومى در شرايط آزمايشكاه Table 1. The effect of different doses of gamma radiation on germination of landrace rice varieties in the laboratory

\begin{tabular}{|c|c|c|c|c|c|c|c|c|}
\hline طول سانتىمتر) & طول ريشتهر) & جوانهزنى & جوانهزنى درصد & جوانهزنى & شاخص بنيه بذر & جوانهزنى & & دز يرتو كاما (كرى) \\
\hline $4 / 1 q^{a}$ & $r / \Delta \Delta^{a}$ & $\Lambda \varepsilon / \Lambda)^{a}$ & $11 / 19^{c}$ & $T F / q \mu^{a}$ & $\Delta N / r \omega^{a}$ & $9 N / *^{a}$ & & صفر \\
\hline$r / \wedge r^{\mathrm{a}}$ & $r / \Delta \gamma^{\mathrm{a}}$ & $\Lambda \mu^{\mathrm{a}}$ & $\mid F / T e^{\mathrm{DC}}$ & $r \mu / \Delta \cdot{ }^{a}$ & $\Delta T / / \Gamma^{a^{a}}$ & $q \Upsilon / q \Psi^{\mathrm{aD}}$ & & I.. \\
\hline$r / q^{a b}$ & $r / q^{a b}$ & $V M / \mu Y^{D}$ & $r \mu / 9 g^{\mathrm{ad}}$ & $I V / M^{D}$ & $\mathrm{rN} / \mathrm{FV}^{\mathrm{D}}$ & $\Delta N / \Gamma^{\mathrm{DC}}$ & & r... \\
\hline$r / 9)^{\mathrm{dc}}$ & $1 / 91^{1 \mathrm{c}}$ & $9 D / \mu^{D}$ & $r \cdot / 9)^{a}$ & $\mid \varepsilon / . .^{\mathrm{Dc}}$ & $r q / q F^{D C}$ & $\Delta \Gamma / \Gamma \omega^{\mathrm{ca}}$ & & ro. \\
\hline$r / \cdot \Lambda^{\mathrm{ca}}$ & $1 / \mathcal{M}^{\mathrm{c}}$ & $\varepsilon \Delta / V \varepsilon^{D}$ & $M r / T \Lambda^{a}$ & $\mid \omega / .^{c}$ & r & $P V / D \cdot{ }^{a e}$ & & r.. \\
\hline $1 / 4^{a}$ & $1 / \Delta 9^{c}$ & G & $r r / A r^{a}$ & $\mid F / g \mu^{c}$ & $N / \& 9^{a}$ & $F Y / V D^{\mathrm{e}}$ & & ra. \\
\hline \multicolumn{6}{|c|}{ ميانگين مربعات } & مرجه آزادى & منابع & \\
\hline $9 / 1 r^{m m}$ & $|/ \Delta|^{m+}$ & $\Lambda \cdot 1 / \mu f^{-n}$ & $V Y G / G V^{T T}$ & $\mid Q F / I V^{m}$ & $1991 / \mathrm{VV}^{2 \pi}$ & $V \mid F / q \Lambda^{* \pi}$ & $\Delta$ & دز يرتو \\
\hline$r / 9 T^{\prime \prime m}$ & $11 / r{ }^{* m}$ & $\Lambda \mid \& / V \Delta^{\circ-}$ & $\mid F \wedge F / N F^{\omega-\pi}$ & $r T / 99^{\circ}$ & $\Lambda F r / 19^{m \pi}$ & $q \varepsilon / \mu r^{n s}$ & 1 & رقم \\
\hline$\cdot / 94^{\mathrm{ns}}$ & $1 / \cdot 9^{-\pi}$ & $r / / q r^{n s}$ & $r e / F \Delta^{n s}$ & $1 / / h^{\mathrm{ns}}$ & $\mid 9 Y / q Y^{N \pi}$ & $19 / \sim^{\mathrm{ns}}$ & $\Delta$ & دز يرتو× رقم \\
\hline.$/ F t^{4}$ & r & $9 V / 19$ & SN/TD & $\Gamma / \leftarrow \wedge$ & QH/GQ & $r \mu / M$ & זr & خطا \\
\hline
\end{tabular}

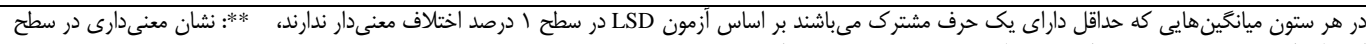

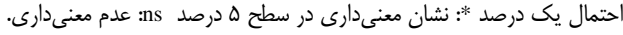

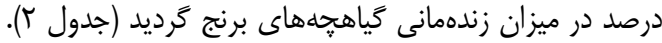

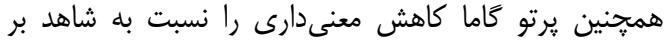

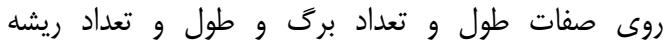

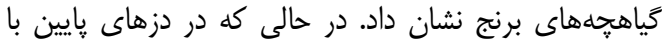

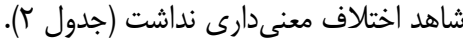

نتايج حاصل از مقايسه ميانگين اثر دزهاى مختلف إنف يرتو

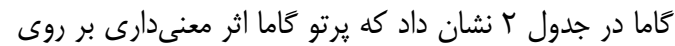

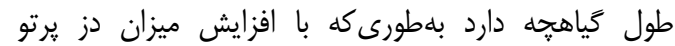

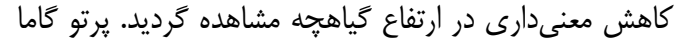

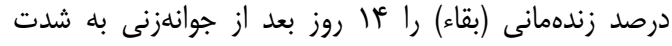

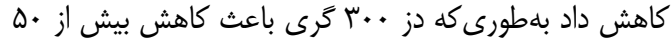


جدول r- اثر دزهاى مختلف يرتو كاما بر صفات مختلف ارقام برنج بومى در شرايط گلخانه

Table 2. The effect of different doses of gamma radiation on the different characteristics of landrace rice varieties in

\begin{tabular}{|c|c|c|c|c|c|c|c|}
\hline 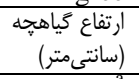 & $\begin{array}{r}\text { درصد بقاء } \\
\text { (درصد }\end{array}$ & 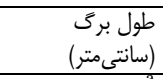 & تعداد برى & (سولتميتر) & تعداد ريشه & & 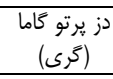 \\
\hline $1 \mathrm{~N} / \mathrm{9}^{\mathrm{a}}$ & $9 \pi / \Delta \cdot \cdot^{2}$ & $\Delta / \Delta \Delta^{a}$ & $\mathrm{r} / \mathrm{l}^{\mathrm{aO}}$ & $1 / 99^{a}$ & $I N / \Delta \gamma^{a}$ & & صفر \\
\hline $10 / 99^{\mathrm{ado}}$ & $\Lambda r / .{ }^{\mathrm{ado}}$ & $r / V^{0}$ & $r / 9 q^{\mathrm{ad}}$ & $N / 90^{\mathrm{a}}$ & $q / r^{a d o}$ & & $1 \ldots$ \\
\hline $\mid F / \Delta \gamma^{\mathrm{ad}}$ & $V \cdot / V D^{D C}$ & $r / F \psi^{\mathrm{C}}$ & $r / / r^{\mathrm{da}}$ & $9 / \Lambda \Delta^{a}$ & $1 . / \% \omega^{\mathrm{ado}}$ & & $r .$. \\
\hline$|1 / 9|^{\mathrm{Dc}}$ & $\Delta V / \Delta \cdot{ }^{c}$ & $T / T^{c}$ & $r / v Q^{a b}$ & $V / V \cdot{ }^{a b}$ & $N / \Delta \gamma^{D C}$ & & ro. \\
\hline$N / F b^{\mathrm{ca}}$ & $f \cdot 1 . .^{a}$ & $r / .9^{\mathrm{c}}$ & $r / \Delta \mathrm{rra}^{\mathrm{ab}}$ & $\Delta / \leftarrow \wedge^{\mathrm{ab}}$ & $V / T V^{D c}$ & & $r .$. \\
\hline$V / \wedge q^{a}$ & $\Gamma / T \Delta^{\mathrm{a}}$ & $1 / 99^{\mathrm{c}}$ & $r /\left.F\right|^{D}$ & $F / 19^{\circ}$ & $8 / .1^{\mathrm{c}}$ & & ra. \\
\hline & & مربعات ميانكين & & & & درجه آزادى & منابع \\
\hline $\mid \pi T / \mathscr{F N}^{N * *}$ & 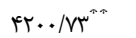 & $\mid V / . T^{\prime}$ &.$/ 9 V^{\circ}$ & 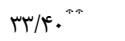 & $F T / F T^{m-T}$ & 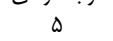 & دز يرتو \\
\hline$\cdot / r \omega^{\mathrm{ns}}$ & $F \cdot N / \pi r^{\text {ns }}$ & $1 / 4 Y^{\mathrm{ns}}$ & $\cdot / \pi \omega^{\mathrm{ns}}$ & $1 / \cdot r^{n s}$ & $\varphi \varphi / 1 \Phi^{m+}$ & 1 & رقم \\
\hline$\Delta / \mathrm{v} \cdot{ }^{\mathrm{ns}}$ & $\Delta \mu / r^{n s}$ & $F / \mu^{\pi * \pi}$ & $\cdot 11 .^{\mathrm{ns}}$ & $1 / \cdot \Delta^{\mathrm{ns}}$ & $r / g v^{n s}$ & $\Delta$ & دز يرتو× رقم \\
\hline$V / q)$ & $10 \cdot / \Lambda \Gamma$ & $\cdot / \Delta F$ &.$/ T \omega$ & $\Delta / 4)$ & سו/ه & سז & خطا \\
\hline
\end{tabular}

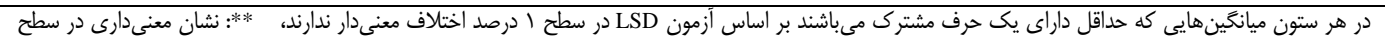

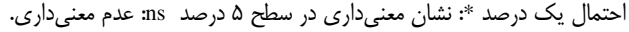

با رقم عنبربو در همين دز تفاوت معنى دارى نشان نداد. علاوه

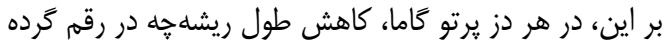

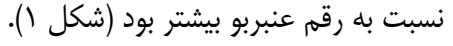

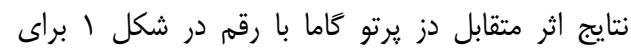
صفت طول ريشه קه كَاهجه

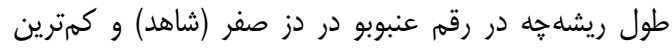

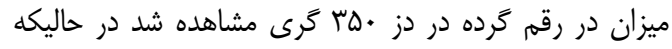

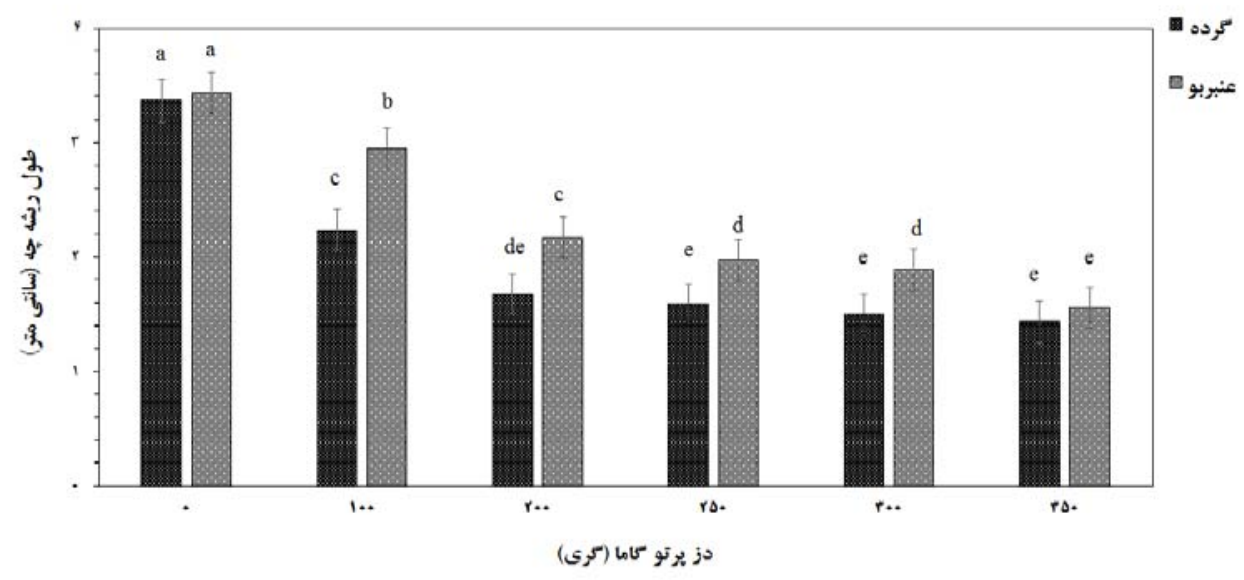

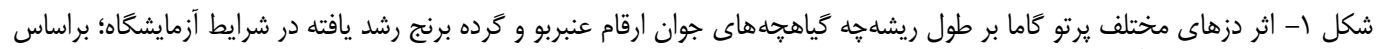

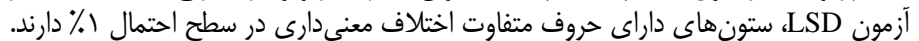

Figure 1. The effect of different doses of gamma irradiation on seedlings root length of Gerdeh and Anbarbo rice varieties in laboratory conditions

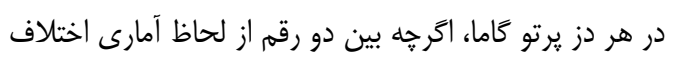

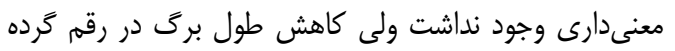

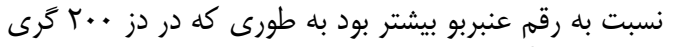

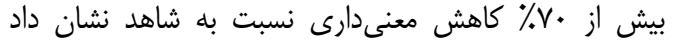

(شكل r) ( )

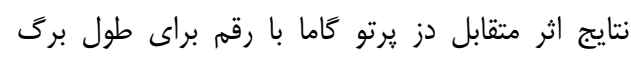

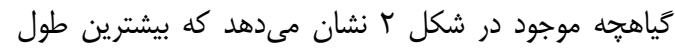

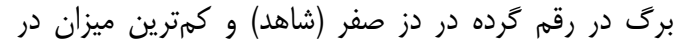

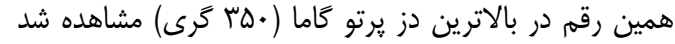
اگر جه با رقم عنبربو در دز مذكور تفاوت معنى دارى نشان ندان 


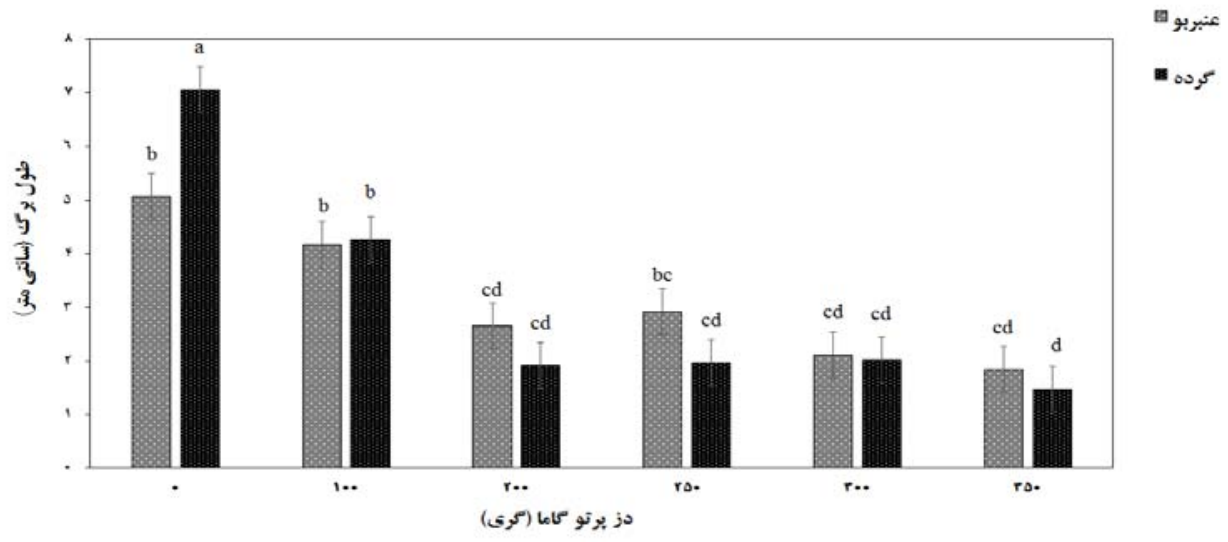

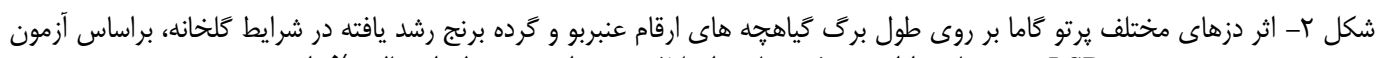
LSD

Figure 2. The effect of different doses of gamma radiation on seedlings root length of Gerdeh and Anbarbo rice varieties in greenhouse conditions

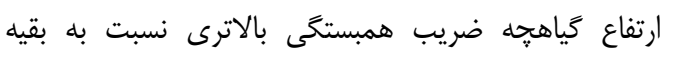

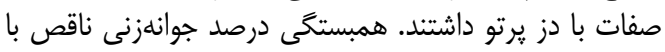
صفات مورد بررسى منفى و معنى دارى بود (جدول دا").
نتايج حاصل از همبستخى صفات نشان داد كه صفات مورد

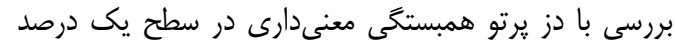

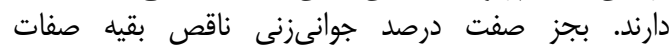

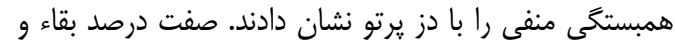

جدول س- همبستخى هاى ساده مؤلفههاى رشد گياهجه و درصد جوانهزنى با دز يرتوتابى در گياه برنج ارقام عنبربو و گرده Table 3. Simple correlation of germination and seedling growth components with the dose of irradiation in Gerdeh and Anbarbo rice varieties

\begin{tabular}{|c|c|c|c|c|c|c|c|c|}
\hline & دز برتو كاما & ارتفاع كَاهجه & درصد بقاء & طول برى & تعداد برى & طول ريشه & تعداد ريشه & درصد جوانهزنى \\
\hline ارتفاع گياهِه & $-\cdot|\Lambda|$ & 1 & & & & & & \\
\hline درصد بقاء & $-\cdot / N \varepsilon^{\prime N T}$ & $\cdot / V e^{-T+}$ & 1 & & & & & \\
\hline طول برى & $-\cdot / V \Psi^{-\pi T}$ & $\cdot / 99^{* \pi}$ &.$|9|^{-\pi}$ & 1 & & & & \\
\hline تعداد برى & - & $\cdot 19 \Lambda^{-1 *}$ & . $/ F T^{N T}$ & . & 1 & & & \\
\hline طول ريشه & $-\cdot / \Delta V^{m-m}$ & $\cdot / v e^{e^{* n}}$ & $-\cdot / \Delta \Delta^{\pi m}$ & $\cdot / q^{* \pi m}$ & $\cdot / V D^{m-n}$ & 1 & & \\
\hline تعداد ريشه & $-* \mid 9 Q$ & $\cdot / N e^{-1}$ & .191 & $\cdot / 49^{\circ}$ & $\cdot 10 \cdot$ & $\cdot \mid \Delta T^{\prime}$ & 1 & \\
\hline درصد جوانهزنى &.$- / 99^{m \pi}$ & $\cdot / \Delta V^{*}$ & $\cdot \mid \Delta \Lambda^{\circ \infty}$ & $\cdot \mid \Delta \Lambda^{* m}$ &.$/ q^{\circ}$ &.$/ 4$ & $.190^{* \cdots}$ & 1 \\
\hline درصد جوانهزنى ناقص & $.190^{m+\pi}$ & $-\cdot \mid \Delta \Gamma^{\omega m}$ & $-\cdot / \Delta r^{\omega *}$ & $-/ \Delta r^{\omega *}$ & $-\cdot / \mu \cdot$ &.$- / \mu^{* \pi}$ &.$- / 109^{* m}$ & $-.190^{-m}$ \\
\hline
\end{tabular}

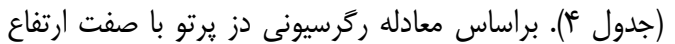

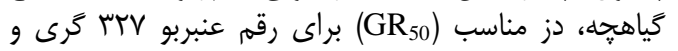

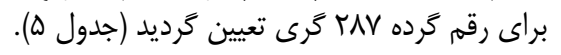

نتايج حاصل از رگرسيون خطى نشان داد كه صفات ارتفاع دراء

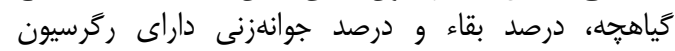

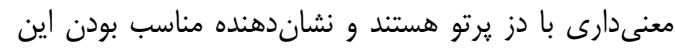

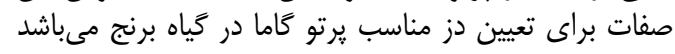

جدول عَ- تجزيه رگرسيون خطى دز يرتو گاما با صفات ارتفاع، درصد بقا و درصد جوانهزنى در ارقام بومى برنج Table 4. Gamma irradiation dose linear regression analysis with height, survival and germination of landrace varieties of rice

\begin{tabular}{|c|c|c|c|c|}
\hline منابع & درجه آزادى & & ميانگين مربعات & \\
\hline & & درصد جوانهزنى & ارتفاع گياهحه & درصد بقاء \\
\hline ركرسيون & 1 & $r \Delta \backslash N / \cdot r^{\top m}$ & $\Delta q T / V T^{\cdots}$ & $r .991 / \omega^{-m}$ \\
\hline باقىمانده & 48 & $\Lambda T / T \Delta$ & $V / I I$ & $g^{\mu} \Delta \Delta / / \Gamma$ \\
\hline كل & iv & & & \\
\hline
\end{tabular}




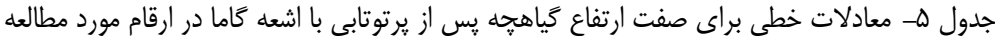
Table 5. Linear equations for the height of seedlings after irradiation with gamma rays in the cultivars

\begin{tabular}{|c|c|c|}
\hline رقم & معادله خطى & دز \\
\hline عنبربو & $Y=-0.0286 x+17.626$ & TTV \\
\hline كر & $Y=-0.032 x+19.048$ & TAV \\
\hline
\end{tabular}

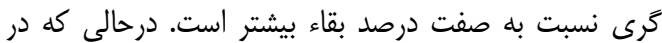

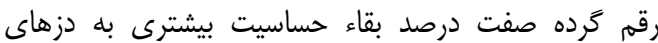

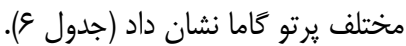

بررسى ميزان حساسيت ارقام برنج به اشعه كاما نشان داده

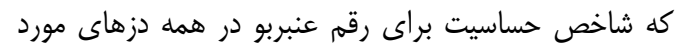

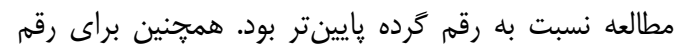

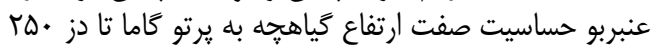

جدول צ- مقايسه ميزان حساسيت ارقام برنج عنبربو و گرده به اشعه گاما براساس شاخص حساسيت به تنش Table 6. Comparison of sensitivity to gamma radiation of Gerdeh and Anbarbo rice varieties on stress susceptibility index

\begin{tabular}{ccccccc}
\hline \multicolumn{7}{c}{} \\
\hline
\end{tabular}

داد كه يرتو كاما باعث كاهش معنى دارى در ميزان رشد، تعداد

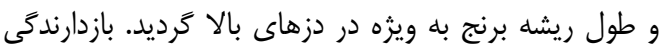

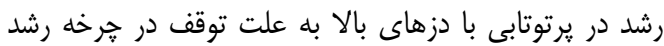

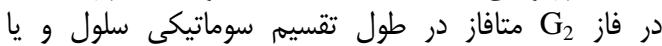

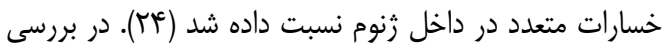

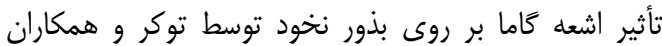
تاهش (TV)

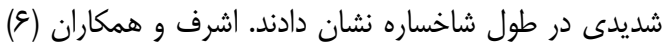

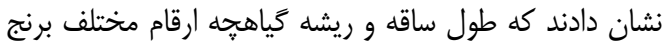

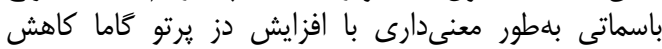

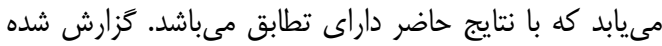

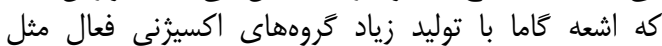

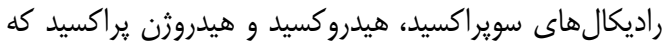

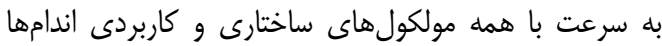

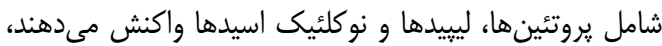

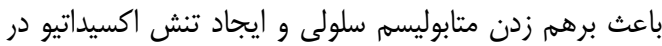

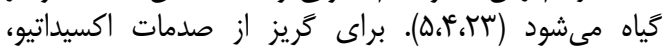

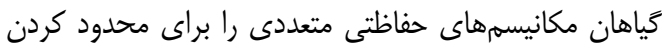

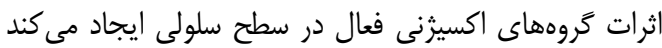

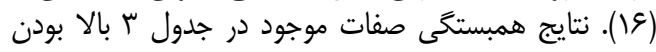

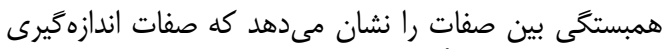

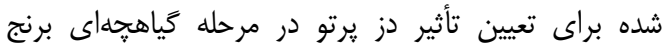

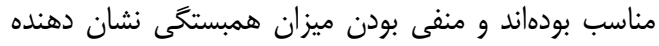

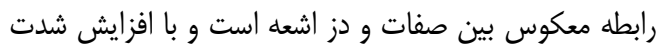

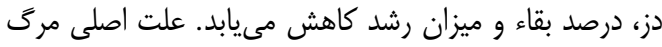

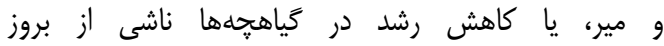
ناهنجارىهاى مختلف در فعاليتهاى متابوليسمى و رشد كَّاه

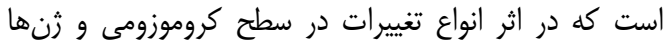

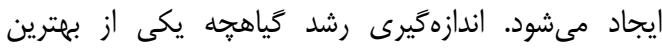

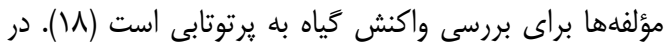

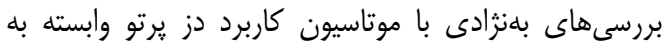

بيشترين كاهش ميزان صفات درصد جوانهزنى، سرعت

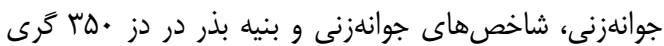

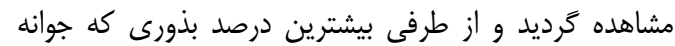

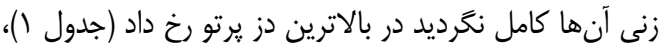

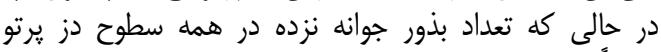

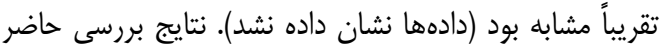

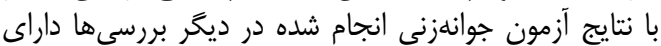

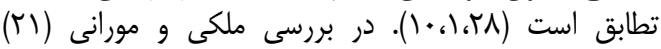

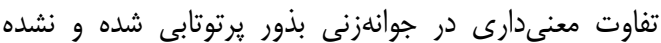

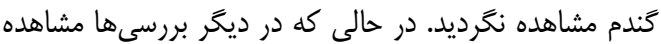

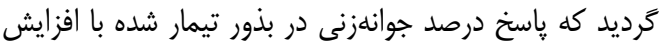

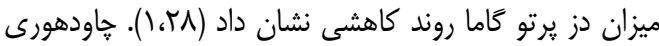

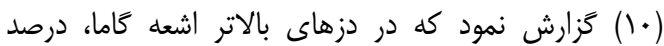

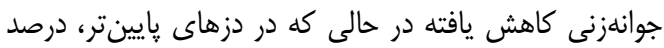

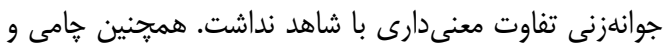

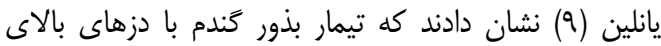

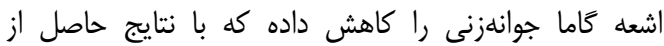

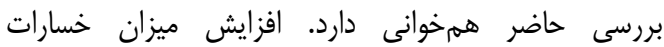

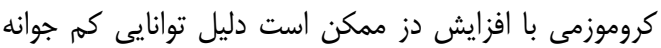

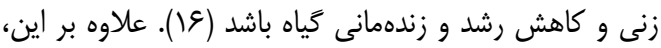

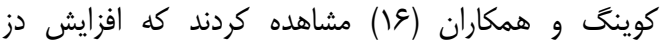

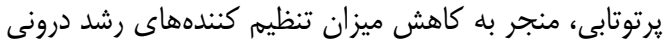

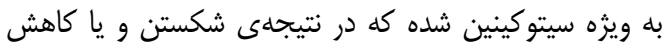

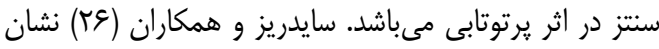

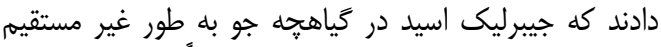

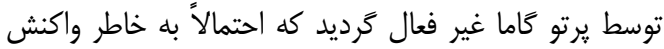

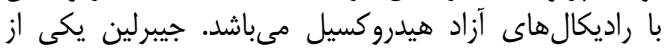

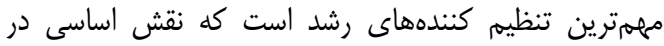

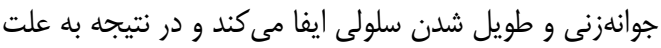

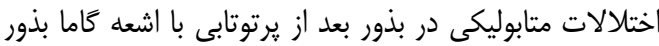

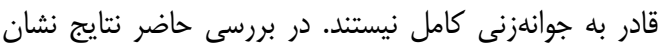


رشد، براى اطمينان از وقوع موتاسيون لازم، كافى و مناسب

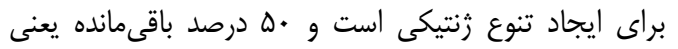

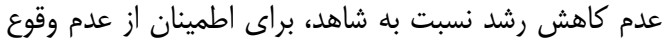

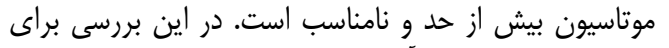

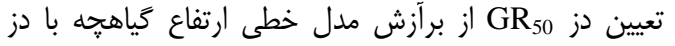

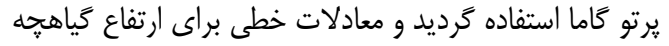

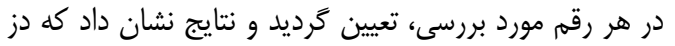

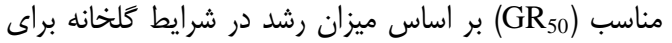

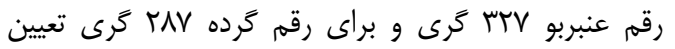

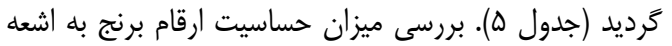

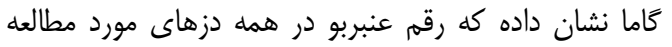

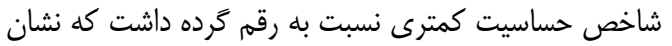

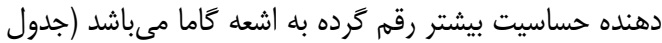

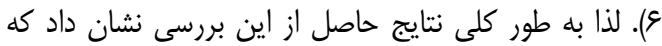

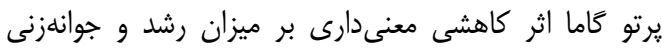

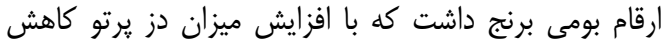

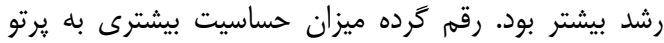

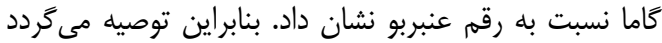

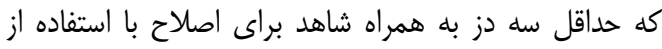

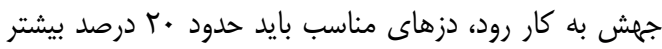

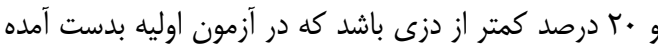

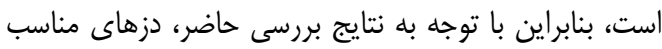

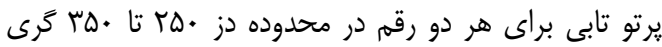
مىباشد.
گَونه گياهى است و مستلزم تعيين دز مناسب با براسى

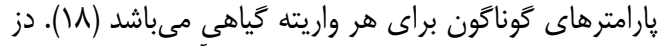

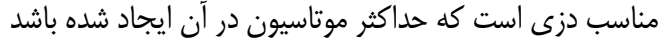
(IV)

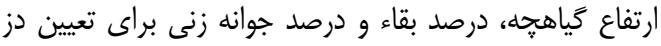

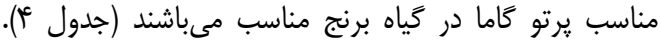

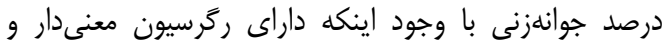

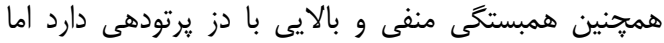

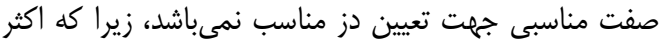

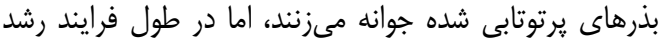

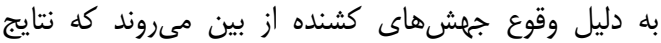

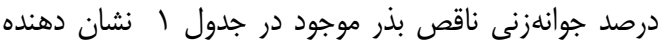

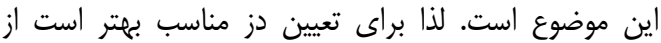

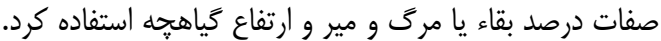
در اين ميان استفاده از دز

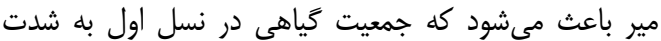

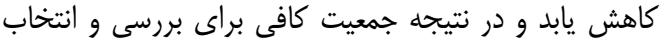

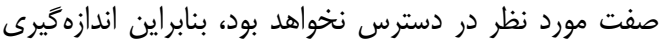

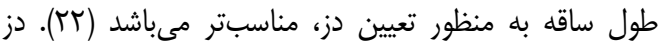

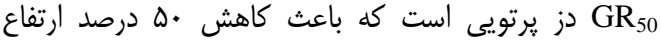

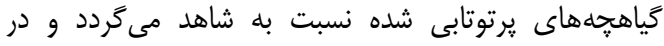

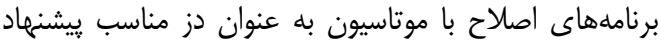

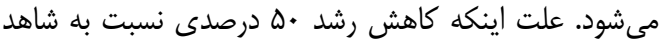

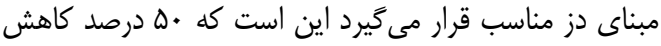



gibberellic acid on germination and alkaloid production in Atropa belladonna. Australian Journal of Basic and Applied Sciences, 2: 401-405.

2. Abdul-Baki, A.A. and J.D. Anderon. 1973. Vigor determination in soybean by multiple criteria. Crop Science, 13: 630-633.

3. Allard, R.W. 1996. Genetic basis of the evolution of adaptedness in plants. Euphytica, 92:1-11.

4. Al-Rumaih, M.M. and M.M. Al-Rumaih. 2008. Influence of ionizing radiation on antioxidant enzymes in three species of Trigonella. American Journal of Environmental Sciences, 4: 151-156.

5. Ashraf, M. 2009. Biotechnological approach of improving plant salt tolerance using antioxidants as markers. Biotechnology Advances, 27:84-93.

6. Ashraf, M., A.A. Cheema, M. Rashid and Z. Qamar. 2003. Effect of gamma rays on $\mathbf{M}_{1}$ generation in Basmati rice. Pakistan Journal of Botany, 35: 791-795

7. Babaei, A.R. 2010. Radio sensivity studies of morpho-physiological characteristics in some Iranian rice varieties (Oryza sativa L.), In $\mathrm{M}_{1}$ generation. African Journal of Agricultural Research, 5: 21242130.

8. Baloch, A.W., A.M. Soomro, G. Mustafa, M.S. Bughio and H.R. Bughio. 1999. Mutagenesis for reduced plant height and high grain yield in Jajai 77, aromatic rice (Oryza sativa L.) variety. Pakistan Journal of Botany, 31: 469-474.

9. Chaomei, Z. and M. Yanlin. 1993. Irradiation induced changes in enzymes of wheat during seed germination and seedling growth. Acta Agriculturae Nucleatae Sinica, 7: 93-97.

10. Chaudhuri, K.S. 2002. A simple and reliable method to detect gamma irradiated lentil (Lens culinaris Medik.) seeds by germination efficiency and seedling growth test. Radiation Physics and Chemistry, 64: 131-136.

11. Ellis, R.H. and E.H. Roberts. 1981. The quantification of ageing and survival in orthodox seeds. Seed Science and Technology, 9: 377-409.

12. Fischer, R.A. and R. Maurer. 1978. Drought resistance in spring wheat cultivars. I. Grain yield response. Australian Journal of Agricultural Research, 29: 897-912.

13. Ghannadha, M.R. 1988. A study on the sensitivity of species and varieties of cereals and legumes to different doses of gamma rays. MSc Thesis, $155 \mathrm{pp}$.

14. Hameed, A., T.M. Shah, M.B. Atta, M.A. Haq and H. Sayed. 2008. Gamma irradiation effects on seed germination and growth, protein content, peroxidase and protease activity, lipid peroxidation in Desi and Kabuli chickpea. Pakistan Journal of Botany, 40: 1033-1041.

15. Heal, G., B. Walkerb, S. Levinc, K. Arrowg, P. Dasguptae, G. Dailyd, P. Ehrlichd, K.G. Malerf, N. Kautskyh, J. Lubchencoi, S. Schneiderd and D. Starrettg 2004. Genetic diversity and interdependent crop choices in agriculture. Resource and Energy Economics, 26: 175-184

16. Kiong, A., A. Ling Pick, S.H. Grace Lai and A.R. Harun. 2008. Physiological responses of Orthosiphon stamineus plantlets to gamma irradiation. American-Eurasian Journal of Sustainable Agriculture, 2: 135-149.

17. Kovacs, E. and A. Keresztes. 2002. Effect of gamma and UV-B/C radiation on plant cells. Micron, 33: 199-210.

18. Majd, P. and M. Ardekani. 2010. Nuclear techniques in agricultural sciences. 3th edn, Inc. Tehran University, $384 \mathrm{pp}$.

19. Majidi, Z., N. Babaeian-Jelodar, G. Ranjbar, N. Bagheri. 2013. Study of induced variation by ethyl methane sulphonate and sodium azide on Tarrom Mahali rice cultivar. Journal of Crop Breeding, 5(12): 49-61. (In Persian)

20. Mba, C., R. Afza, S.M. Jain, G.B. Gregorio and F.J. Zapata-Arias. 2007. Induced mutations for enhancing salinity tolerance in rice. In: Jenks, M.A., P.M. Hasegawa, S. Mohan Jain (eds.) Advance in molecular breeding toward drought and salt tolerant crops, pp: 413-454.

21. Melki, M. and A. Marouani. 2009. Effects of gamma rays irradiation on seed germination and growth of hard wheat. Environmental Chemistry Letters, 8: 307-310.

22. Naserian Khiabani, B., H. Ahari Mostafavi, H. Fathollahi, S. Vedadi and M.A. Mosavi Shalmani. 2008. Suitable gamma ray dose determination in order to induce genetic variation in kaboli chickpea (Cicer Arietinum L.). Journal of Nuclear Science and Technology, 42:19-25

23. Noreen, Z. and M. Ashraf. 2009. Changes in antioxidant enzymes and some key metabolites in some genetically diverse cultivars of radish (Raphanus sativus L.). Environmental and Experimental Botany, 67: 395-402.

24. Preussa, S.B. and A.B. Britta. 2003. A DNA-damage-induced cell cycle checkpoint in Arabidopsis. Genetics, 164: 323-334.

25. SAS Institute. 2003. The SAS system for Windows. Release 9.1. SAS Inst., Cary, NC.

26. Sideris, E.G., M.M. Nawar and R.V. Nilan. 1971. Effect of gamma radiation on gibberellic acid solutions and gibberellin-like substrances in barley. Radiation Botany, 11: 209-214.

27. Toker, C., B. Uzun, H. Canci and F. Oncu Ceylan. 2005. Effects of gamma irradiation on the shoot length of cicer seeds. Radiation Physics and Chemistry, 73: 365-367.

28. Wi, S. G., B.Y. Chung and J.S. Kim. 2007. Effects of gamma irradiation on morphological changes and biological responses in plants. Micron, 38: 553-564.

29. Younesi Hamzeh khanloo, M., A. Izadi Darbandi, N. Pirvali Biravanvand, M.T. Hallajian and A. Majdabadi. 2010. Study of Relationship Between Some Agro-Morphological Traits With Yield in M7 Generation of Soybean Mutant lines Irradiated by Gamma Ray. Journal of Crop Breeding, 2: 30-46. (In Persian). 


\title{
Effect of Gamma Irradiation on Growth and Determine Optimum Dose in Order to Induce Genetic Variation in landrace Rice (Oryza sativa L.) Cultivars
}

\author{
Leila Bagheri $^{1}$, Rahim Amiri-Khah ${ }^{2}$, Mansour Noori ${ }^{2}$ and Kameran mozafari ${ }^{3}$ \\ 1- Researcher, Nuclear Agriculture Research School, Nuclear Science and Technology Research Institute, Karaj- \\ Iran (Corresponding author: lbagheri@ nrcam.org) \\ 2 and 3- Expert and Researcher, Nuclear Agriculture Research School, Nuclear Science and Technology Research \\ Institute, Karaj-Iran \\ Received: June 24, $2015 \quad$ Accepted: November 1, 2015
}

\begin{abstract}
Mutation breeding as an alternative to conventional breeding, is commonly used to improve current varieties and to generate new varieties. Genetic variation in rice is very low, because of its self-pollination. This study, carried out to determine the $\mathrm{GR}_{50}$ dosage for an irradiation mutagenesis study on the rice and to determine the effects of gamma irradiation on germination and growth rate of rice seedlings. The elite seeds of landrace rice CVs. Anbarbo and Gerdeh after moisture regulation (11-13\%) were exposed to 100, 200, 250, 300, and 350 Gy doses of gamma irradiation from ${ }^{60} \mathrm{Co}$ and then grown under greenhouse and laboratory conditions. In both conditions, the experiment was a factorial design laid out in a completely randomized design with four replications. The results showed that the lowest values of germination parameters were observed under 350 Gy gamma irradiation. Also, the results showed that with an increase in gamma irradiation dose, the growth rate of both cultivars of rice decreased. In this respect, the decrease of growth rate has a linear relationship with the gamma irradiation dose. Results demonstrated that with increasing of gamma irradiation dose the plantlet height, survival percentage, the number and length of root and leaf in the both conditions were decreased. Gerdeh variety showed a greater degree of sensitivity to gamma irradiation than the Anbarbo variety. Based on our results, 327 and 287 Gy doses of gamma irradiation were selected for the maximum mutation in Anbarbo and Gerdeh, respectively. Finally, in both cultivars, dosages ranging from 250 to $350 \mathrm{~Gy}$ are recommended as the appropriate dose of irradiation to create genetic variation for mutation breeding programs.
\end{abstract}

Keywords: Gamma Irradiation, Genetic Variation, Mutation, Rice 